CASE REPORT

\title{
Primary Zirconia Crowns: An Era of Esthetic Management in Early Childhood Caries Affected Children
}

\author{
Shrirang Sevekar ${ }^{1}$, Ashwini Avanti $^{2}$, Mihir N Jha $^{3}$, Laresh Mistry ${ }^{4}$, Varsha Patel ${ }^{5}$, Sujata Hirave ${ }^{6}$
}

\begin{abstract}
Aim and objective: To report the case series of zirconia crown rehabilitation in grossly carious primary teeth.

Background: Early childhood caries (ECC) pose restorative as well as esthetic concerns. Multiple options for extracoronal restorations like stainless steel crown, polycarbonate crown, and strip crown are presently available. Recently, the zirconia crown has become a good alternative due to its excellent esthetic quality, greater strength, and ease of placement.

Case description: This case report presents a series of grossly carious primary maxillary incisors and mandibular molars restored with preformed zirconia crowns. The treatment describes a simple and effective means of restoration of grossly carious primary teeth.

Conclusion: Zirconia crowns are simple and effective means in the rehabilitation of grossly carious, badly broken down, and fractured primary anterior and posterior teeth.
\end{abstract}

Keywords: Dental caries, Esthetics, Zirconium.

Journal of Contemporary Dentistry (2019): 10.5005/jp-journals-10031-1276

\section{BACKGROUND}

Early childhood caries (ECC) is defined as the presence of one or more decayed (non-cavitated or cavitated lesions), missing (due to caries), or filled tooth surfaces in any primary tooth in a child under the age of $6 .{ }^{1}$ It is a multifactorial disease that involves those teeth which are considered immune to decay. The badly brokendown primary teeth can precede severe socio-behavioral issues and further complex dental problems in infants and toddlers. ${ }^{2,3}$ Previously, extraction was the treatment of choice for such cases. These led to poor esthetics, compromised mastication, and multiple habit developments. ${ }^{2}$ Numerous esthetic restorative materials have been utilized to restore these severely decayed primary anterior teeth. Materials like resin-modified glass ionomer (RMGIC), compomer, and resin composite have been used to strengthen these structurally weakened teeth with limited success. Full coronal esthetic restorations like composite strip crowns (CSC), preveneered stainless steel crowns (PVSSC), and open-faced stainless steel crowns (SSCs) have been used with satisfactory results. Over a while, parents are expecting higher esthetic standards with a demand for natural-looking restorations. Dental professionals often experience parental keen involvement in clinical decision-making of anterior esthetic restorations. Recently introduced prefabricated primary zirconia crown has attracted a lot of attention among researchers and patients as an alternative to PVSSC, SSC, and CSC. ${ }^{4}$

The purpose of this case report was to explain the clinical steps in the rehabilitation of grossly carious primary maxillary incisors and mandibular primary molar in young children respectively using primary zirconia crowns.

\section{Case Descriptions}

\section{Case 1}

A 4-year-old boy reported to the Department of Pediatric and Preventive Dentistry with the chief complaint of severely decayed primary upper front teeth. There was no relevant medical history. \begin{tabular}{l}
\hline${ }^{1-6}$ Department of Pediatric and Preventive Dentistry, MGM Dental \\
College and Hospital, Navi Mumbai, Maharashtra, India \\
Corresponding Author: Ashwini Avanti, Department of Pediatric and \\
Preventive Dentistry, MGM Dental College and Hospital, Navi Mumbai, \\
Maharashtra, India, Phone:+91 7019277207, e-mail:ashwinikorlahalli@ \\
gmail.com \\
How to cite this article: Sevekar S, Avanti A, Jha MN, et al. Primary \\
Zirconia Crowns: An Era of Esthetic Management in Early Childhood \\
Caries Affected Children. J Contemp Dent 2019;9(3):150-154. \\
Source of support: Nil \\
Conflict of interest: None
\end{tabular}

The patient gave a history of nocturnal pain in the upper front region in the last 1 month. The pain was spontaneous, dull, localized in the maxillary incisor region. Intraoral examination revealed the presence of fully erupted all the primary teeth with grossly carious $51,52,61,62$ (Fig. 1A). The radiographic examination confirmed the pulpal involvement of maxillary incisors. A final diagnosis of chronic irreversible pulpitis in relation to $51,52,61,62$ was made based on pain history, clinical findings, and radiographic interpretation. Diet counseling, oral prophylaxis, and topical fluoride application were carried out. Pulpectomy was planned for these teeth followed by esthetic rehabilitation. Zirconia crown (Kinder Krowns, St Louis Park, MN 55426, USA) placement was decided after pulpectomy for all the maxillary incisors after thorough discussion and express consent from parents. Accordingly, zirconia crowns were placed initially on 51, 61 (Fig. 1B) followed by crown placement on 52 and 62 in subsequent appointments (Fig. 1C). Follow-up was done after 1 month with satisfactory gingival adaptation to zirconia crowns (Fig. 1D).

\section{Case 2}

A 4-year-old girl reported to the department with the chief complaint of severely decayed lower left first primary molar. 

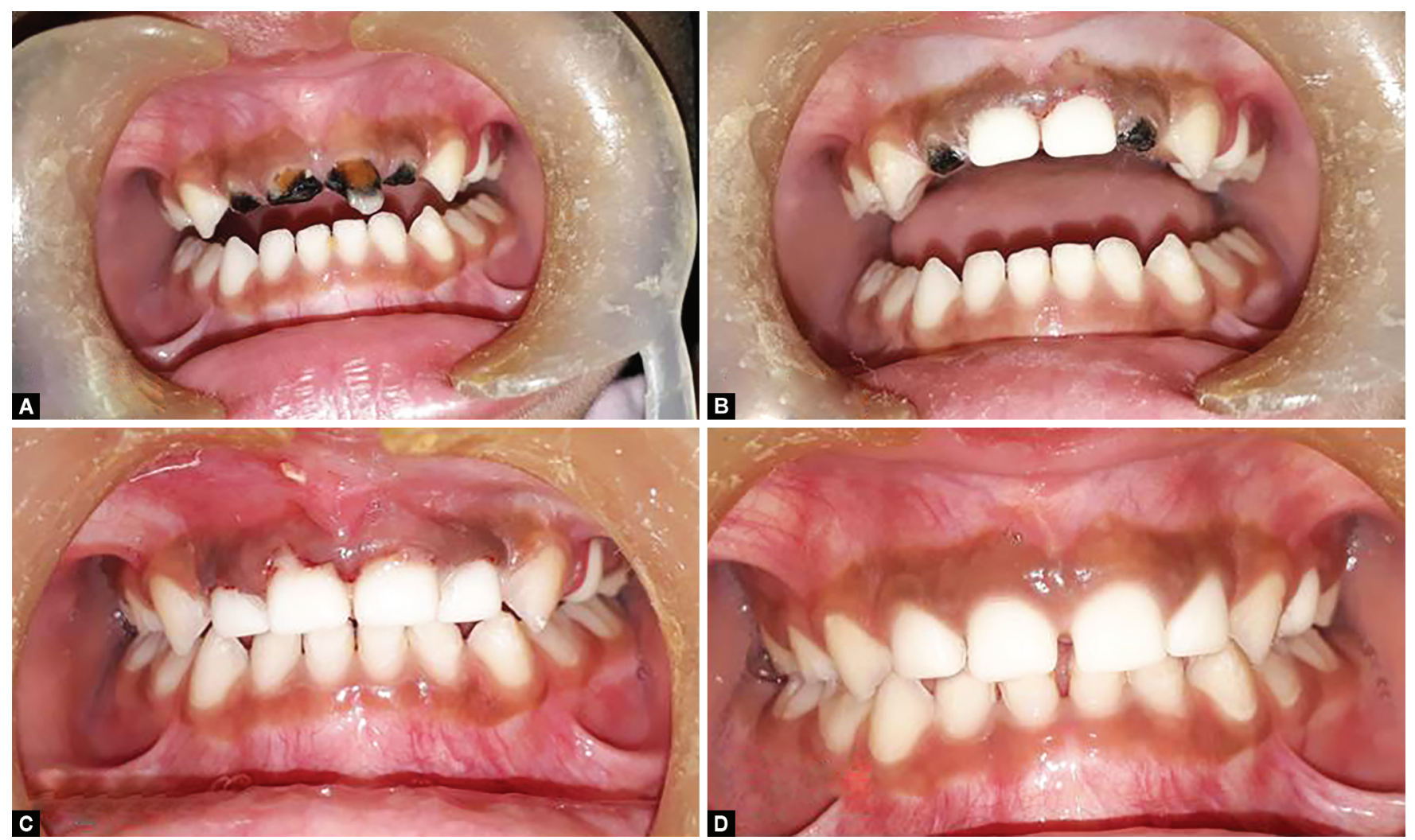

Figs 1A to D: (A) Preoperative grossly carious 51, 52, 61, 62; (B) Postoperative zirconia crown cementation with 51, 61; (C) Postoperative zirconia crown cementation with 51, 51, 61, 62; (D) One-month follow-up

There was no relevant medical history. The patient gave a history of nocturnal pain in the lower left back region of the jaw for 1 month. The pain was gradual, dull, localized in the lower left back region. Intraoral examination revealed a grossly carious 74 . The radiographic examination confirmed the pulpal involvement of the lower mandibular molar. A final diagnosis of chronic irreversible pulpitis in relation to 74 was made based on pain history, clinical findings, and radiographic interpretation. Diet counseling, oral prophylaxis, and topical fluoride application were carried out after taking parent consent. Pulpectomy was planned for this tooth followed by esthetic rehabilitation (Fig. 2A). Zirconia crown (Kinder Krowns, St Louis Park, MN 55426, USA) was placed after thorough discussion and express consent from parents. Zirconia crown was placed on 74 (Figs $2 \mathrm{~A}$ and $\mathrm{C}$ ). Follow-up was done at regular intervals.

\section{Case 3}

A 5-year-old boy reported to the department with the chief complaint of a badly broken lower right primary tooth. There was no relevant medical history. The patient gave a history of nocturnal pain in the lower right region for the last 1 month. The pain was spontaneous, dull, localized in the lower right back region. Intraoral examination revealed a grossly carious 85 . The radiographic examination confirmed the pulpal involvement of the tooth. A final diagnosis of chronic irreversible pulpitis in relation to 85 was made based on pain history, clinical findings, and radiographic interpretation. Diet counseling, oral prophylaxis, and topical fluoride application were carried out after taking parental consent. Pulpectomy was planned (Fig. 3A) for this tooth followed by esthetic rehabilitation of zirconia crown (Kinder Krowns, St Louis Park, MN 55426, USA) (Fig. 3F). Follow-up was done at regular intervals.

\section{Steps in Tooth Preparation}

Preselection of appropriate crown size is always advised before tooth preparation for a better outcome. This ensures a beautiful and natural restoration consistent with the child's original smile.

\section{Crown Selection}

The appropriate size of the crown can be decided using try-in crowns before initiating tooth reduction. Crown is selected based on the mesiodistal dimension of the noncarious tooth. Correct crown selection is especially critical in cases with tight contacts, crowding, or loss of mesiodistal space.

\section{Preparation of the Tooth}

The most important aspect for crown placement is adequate tooth preparation. Good preparation will essentially lead to excellent crown fit. Subsequently, this will save a lot of chairside operating time. The preparation should be such that the crown fits passively without any extrapressure during seating. These crowns may need a greater amount of circumferential tooth reduction than SSCs. Appropriate pulp protection needs to be provided to reduce the chances of future pulpal pathology.

- Incisal/occlusal, proximal, and supragingival reduction

- Reduction of the incisal length/occlusal length should be approximately 1.5-2 mm. This helps in a suitable thickness of zirconium for better esthetics. The preparation should follow the natural occlusal/incisal contours of the tooth (Fig. 3B). The 

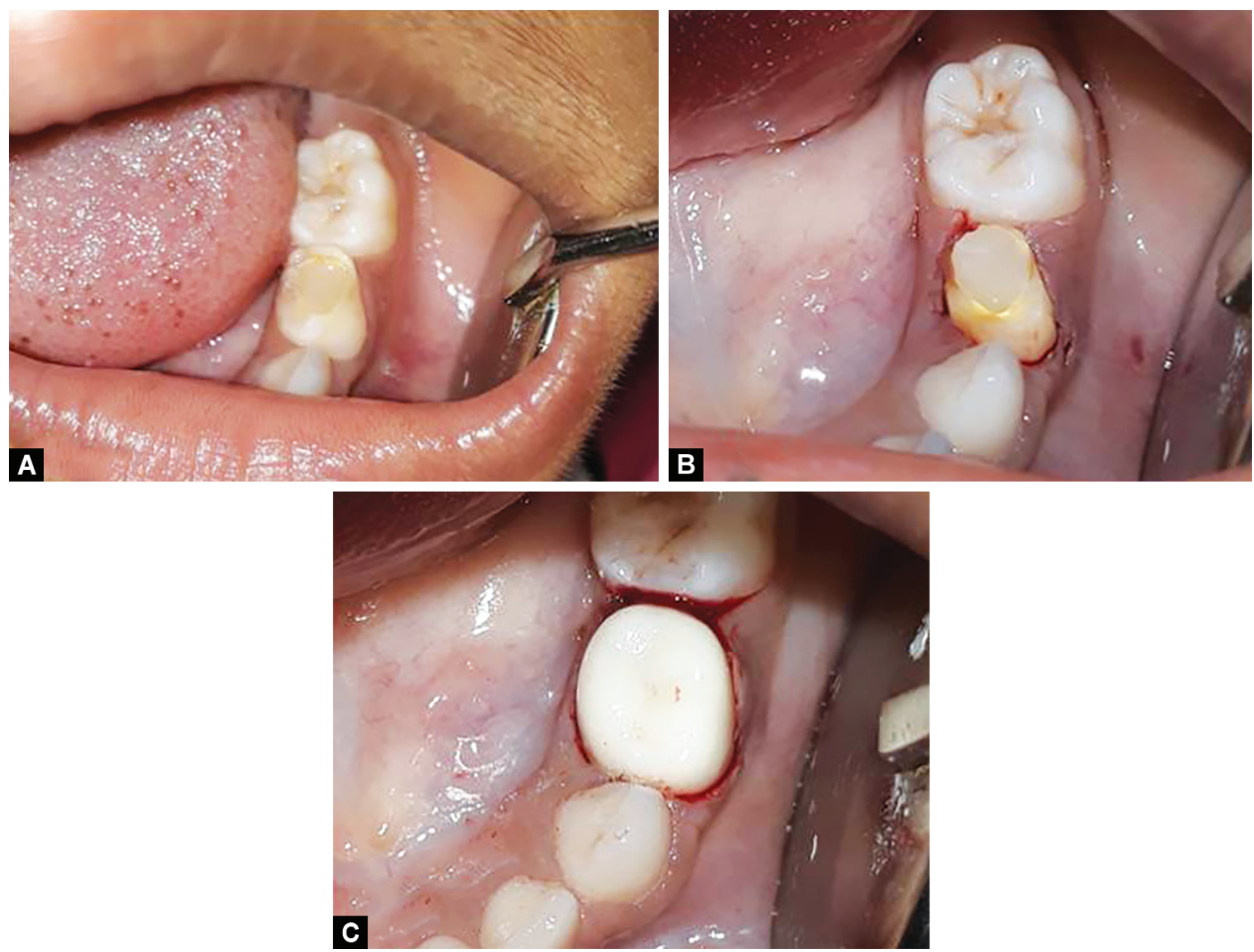

Figs $2 \mathrm{~A}$ to C: (A) Preoperative pulpectomized 74; (B) Completed crown preparation before the cementation i.r.t 74; (C) Postoperative zirconia crown cementation i.r.t 74
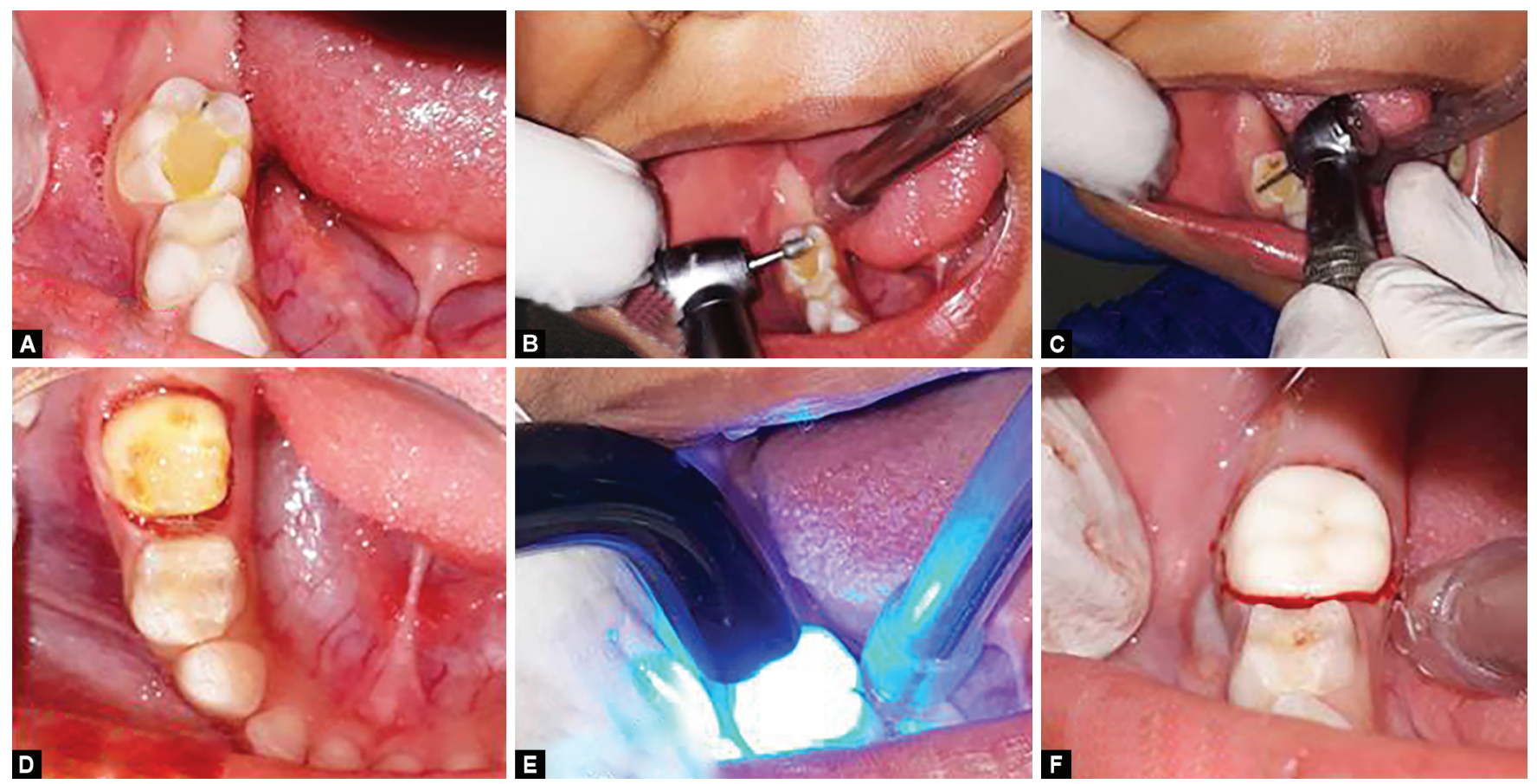

Figs 3A to F: (A) Preoperative pulpectomized 85; (B) Occlusal reduction of 85; (C) Circumferential/buccal reduction of 85; (D) Completed crown preparation before the cementation i.r.t 85; (E) Cementation with light curing; (F) Postoperative zirconia crown i.r.t 85

incisal reduction has to be adequate to ensure clearance in protrusive movements of the mandible.

- Interproximal contacts should be broken first. The proximal reduction is accomplished to maintain parallelism between mesial and distal line angles. Maximum precaution should be taken to prevent any damage to the adjacent teeth. An adequate proximal reduction will allow the zirconia crown to fit passively.

- The circumferential tooth reduction (buccal/labial/lingual) should be around $20-30 \%$ or $0.5-1.25 \mathrm{~mm}$ as necessary. A 
gradual reduction should follow facial and lingual contours and planes of the tooth (Fig. 3C).

- These steps are performed using course tapered diamond (\#5855-012) or carbide burs (\#330) a coarse football diamond bur (\#5368-023) to reduce the incisal/occlusal surface teeth.

- Subgingival reduction

- The prepared margin should be maintained feather-edge 1-2 mm subgingivally on all surfaces. This ensures healthy gingival adaptation and maximum retention.

- Excessive tooth reduction should be avoided in the cervical areas for adequate crown retention. Subgingival tooth reductions should be done with a thin, tapered diamond bur (\#134F-014) to prevent tissue laceration.

\section{Final Preparation of the Crown}

- Final preparation involves rounding of line angles and point angles (Fig. 3D).

- Care must be taken to prevent any formation of undercuts or ledges subgingivally.

\section{Crown Trial}

- Method 1: Trial crowns provided by the manufacturer can be placed on the prepared tooth to see the passive fit. These crowns can be reused after appropriate disinfection.

- Method 2: Preselected zirconia crowns placed directly on the prepared tooth. If the crown gets contaminated with saliva or blood, cleaning of the internal crown surface should be done with extraoral cleaning paste (Ivoclean, Ivoclar Vivadent AG, Bendererstrasse 29494 Schaan Liechtenstein) before cementation.

Zirconium crowns should fit subgingivally without distorting the gingival tissue. A smaller crown size should be selected if the gingival contour is not natural. An additional tooth reduction should be performed if necessary. For multiple crown placements, all crowns are tried together to confirm a passive fit on the prepared teeth.

\section{Zirconia Crown Adjustment}

- Zirconium crowns should only be adjusted with burs specifically designed for adjusting zirconia restorations.

- Adjustment burs can be used to circumferentially reduce and feather the crown margins. No other areas of the crowns should be adjusted.

- When any adjustments are made, the zirconium crown polishers should be used to restore a smooth surface.

\section{Crown Cementation}

- The gingival bleeding should be controlled before cementation with the help of any hemostatic agent.

- Resin cement (BioCem Universal BioActive cement, Ivoclar Vivadent AG, Bendererstrasse 29494 Schaan Liechtenstein), or light-cured RMGIC (GC Gold Label, GC corporation, 3-2-14 Hongo, Bunkyo-Ku, Tokyo 113-0033, Japan) can be used to cement zirconia crowns (Fig. 3E). For self-cure cement, the crown has to be held firmly till the cement sets. Excess cement should be cleaned (Fig. 3F) and occlusion should be checked for high points. Accordingly, the opposing teeth should be adjusted as necessary. ${ }^{5}$

\section{Discussion}

Esthetic management of grossly decayed or traumatized primary teeth in children is a very challenging task. Small size, high placement of pulp horns, thinner enamel, and reduced surface area for bonding possess a great challenge. The unpredictable behavior of the children further complicates the management. ${ }^{6}$ Though there is inadequate research on the impact of unesthetic restoration in primary teeth on psychosocial development in children, optimal esthetics in anterior teeth should be a clear treatment goal in present times. $^{7}$

Many restorative materials are available for treating grossly carious primary teeth, such as, GIC, compomers, composites, etc. Nonetheless, they have shown limited clinical success. The inadequate clinical evidence on the superiority of one restoration over another further complicates their selection. These teeth often need full coverage restorations for long term satisfactory outcome. Multiple factors, such as, parental esthetic demand, child's unpredictable behavior, and moisture/hemorrhage control should be considered before finalizing the type of full-coverage restoration. These factors can affect the long-term prognosis and outcome of the treatment. ${ }^{8}$

There are multiple full coronal restorations available commercially for the clinicians to restore badly broken anterior and posterior primary teeth. These semipermanent restorations need to be esthetically pleasing, resilient, and retentive. For a long time, SSCs were the most viable and convenient option outperforming amalgam and direct composites. ${ }^{9}$ However, the unesthetic appearance of these crowns makes it the least favored choice for the patient. The simple alteration of SSC with bonded resin veneers has shown definite promise as an esthetic alternative to full coverage SSC. ${ }^{8}$ Though this modification is resilient, the visibility of the metal margin around pre-veneered resin remains a serious drawback. ${ }^{10}$

Bonded celluloid strip crowns are another full coverage restorative option for the management of carious primary incisors. These crowns are a highly esthetic, natural-looking well-accepted alternative. But they have the inherent drawback of technique sensitivity and color instability. Even under ideal conditions, strength and wear resistance are inferior to the SSCs. ${ }^{11}$ A higher failure rate is reported due to inappropriate patient selection, lack of moisture, and hemorrhage control during adhesive application and composite placement. ${ }^{12}$ Furthermore, Dr Lee in his review on the restoration of primary anterior teeth suggested that survival of the strip crown depends on the availability of sound tooth structure. ${ }^{12}$ In our case, the strip crown was not attempted as the teeth were grossly destructed.

Another alternative is the biological crowns of extracted primary teeth. These biological restorations are esthetic and least technique sensitive. The limitation of this procedure includes the setting of the tooth bank, consent from the donor and recipient to use the tooth. The stringent cross-infection control policies of present times have made this technique non-viable. ${ }^{6}$

Zirconia (ceramic steel), a crystalline dioxide of zirconium, is milled from a single densely sintered block of crystal. They are commonly used for the preparation of crowns, fixed partial denture, 
implant abutment, inlay, and onlays. The zirconia crown is highly esthetic, biocompatible, translucent, and much stronger than the porcelain fused metal (PFM) crowns. They are thermostable with low heat conductivity and have a stable chemical resistance with better tolerance to cyclical stresses. ${ }^{13}$ Furthermore, the preformed zirconia crown reduces chairside time and eliminates laboratory fabrication. The zirconia crowns are available commercially in multiple sizes for a better fit.

In our case, we selected a zirconia crown over an open-faced SSC to omit the additional preparation of the labial surface. Moreover, the esthetic appearance of zirconia was far more superior to the open-faced SSC. We observed that zirconia provides superior gingival tissue adaptation. Unlike CSC and SSC, the smooth polished surface of zirconia prevents staining and plaque accumulation. An equilibrium of strength, precision, and translucency permits zirconium restorations to accommodate various clinical situations. ${ }^{14}$

However, we stumbled upon a few disadvantages of the zirconia crown. The need for extensive supra and subgingival tooth preparation (reduction) can sometimes lead to a complication on the health of pulpal and gingival tissues. Compromised pulp or excessive gingival bleeding and eventual recession are not agreeable outcomes for patients or clinicians look forward to. Another disadvantage of these preformed crowns is that they cannot be adjusted, trimmed, or modified-like SSCs. This makes the procedure more tedious and lengthy demanding greater expertise from a clinician with additional cooperation from child patients.

Although the zirconia crown technique is relatively simple, there is low evidence of a long-term success rate available in the literature. This is probably due to the relatively recent launch and less availability of zirconia crowns to clinicians. There is an explicit scope for a prospective long-term study on stability and fracture resistance of the zirconia crowns in the future.

\section{Conclusion}

Within the limitation of the cases described, we conclude that zirconia crowns are simple and effective means in the rehabilitation of grossly carious, badly broken down, and fractured primary anterior and posterior teeth. They have shown unparalleled advantages of improved esthetics and microstructure.

\section{References}

1. American Academy on Pediatric Dentistry, American Academy of Pediatrics. Policy on early childhood caries (ECC): classifications, consequences, and preventive strategies. Pediatr Dent 20082009;30(7 Suppl):40-43.

2. Davies GN. Early childhood caries--a synopsis. Community Dent Oral Epidemiol 1998;26(1 Suppl):106-116. DOI: 10.1111/j.1600-0528.1998. tb02102.x.

3. Jose B, King NM. Early childhood caries lesions in preschool children in Kerala, India. Pediatr Dent 2003;25(6):594-600.

4. Salami A, Walia T, Bashiri R. Comparison of parental satisfaction with three tooth-colored full-coronal restorations in primary maxillary incisors. J Clin Pediatr Dent 2015;39(5):423-428. DOI: 10.17796/10534628-39.5.423.

5. Technical Guide Instructions for Use and General Information. NusmileZR zirconia, https://www.nusmile.com/Plugins/Widgets. FAQ.Vinformatix/Content/FAQ/FAQCategoryFiles/IFU\%2 005\%20 ZR\%20Technical\%20Guide\%20(Eng)\%20Rev\%208.pdf, accessed oct 2020.

6. Ashima G, Sarabjot KB, Gauba K, et al. Zirconia crowns for rehabilitation of decayed primary incisors: an esthetic alternative. J Clin Pediatr Dent 2014;39(1):18-22. DOI: 10.17796/jcpd.39.1.t6725r 5566u4330g.

7. Judd PL, Casas MJ. Psychosocial perceptions of premature tooth loss in children. Ont Dent 1995;72(8):16-18, 20, 22-23.

8. Waggoner WF. Anterior crowns for primary anterior teeth an evidence based assessment of the literature. Eur Arch Paediatr Dent 2006;7(2):53-57. DOI: 10.1007/BF03320815.

9. Croll TP, Helpin ML. Preformed resin-veneered stainless steel crowns for restoration of primary incisors. Quintessence Int 1996;27(5):309_ 313.

10. MacLean JK, Champagne CE, Waggoner WF, et al. Clinical outcomes for primary anterior teeth treated with preveneered stainless steel crowns. Pediatr Dent 2007;29(5):377-381.

11. Waggoner WF. Restoring primary anterior teeth. Pediatr Dent 2002;24(5):511-516.

12. Lee JK. Restoration of primary anterior teeth: review of the literature. Pediatr Dent 2002;24(5):506-510.

13. Al-Amleh B, Lyons K, Swain M. Clinical trials in zirconia: a systematic review. J Oral Rehabil 2010;37(8):641-652. DOI: 10.1111/j.13652842.2010.02094.x.

14. Chang YY. Maximizing esthetic results on zirconia-based restorations. Gen Dent 2011;59(6):440-445. 